\title{
Effect of inhaled corticosteroids on bone mineral density in patients with asthma
}

\author{
Hiroyoshi Watanabe, ${ }^{1}$ Kumiya Sugiyama, ${ }^{1,3}$ Naotatsu Otsuji, ${ }^{1}$ Kentaro Nakano, ${ }^{1}$ Hajime Arifuku, ${ }^{1}$ Tomoshige Wakayama, ${ }^{1}$ \\ Shingo Tokita, ${ }^{1}$ Kenya Koyama, ${ }^{1}$ Hirokuni Hirata, ${ }^{1}$ Masafumi Arima, ${ }^{2}$ Kazuhiro Kurasawa, ${ }^{2}$ Yasutsugu Fukushima ${ }^{1}$
}

\begin{abstract}
Background: Inhaled corticosteroids (ICS) are a safe treatment for asthma. However, at higher doses, ICS use has been reported to inhibit adrenocortical function.
\end{abstract}

Objective: This study aimed to evaluate the effect of ICS on bone mineral density (BMD) in adult patients with asthma.

Methods: Ultrasonic bone densitometry was performed in 40 patients (14 men, 26 women, mean age 61.2 years, mean duration of asthma 6.19 years) who were receiving ICS for asthma, and the whole bone density, thickness of cortical bone, and density of cancellous bone of the radius was measured. The age-matched mean was set as $100 \%$. Lifetime cumulative dose of ICS was calculated using all past prescriptions.

Results: No significant correlations were observed between lifetime cumulative ICS dose and whole bone density $\left(r^{2}=0.011\right)$, cortical bone thickness $\left(r^{2}=0.022\right)$, and cancellous bone density $\left(r^{2}=0.004\right)$. No significant differences were observed between lower and higher lifetime cumulative ICS dose among these BMD parameters (104\% vs $97 \%$, $103 \%$ vs $99 \%$, and $106 \%$ vs $91 \%$, respectively). No significant correlations or differences in lifetime cumulative ICS dose were observed by asthma severity, asthma duration, and pulmonary function. Also, serum markers of bone metabolism showed no significant correlations or differences with lifetime cumulative ICS dose.

Conclusions: In the entire study population, long-term ICS use was safe and was not associated with an increased risk of osteoporosis.

Key words: Asthma, Bone mineral density, Inhaled corticosteroids (ICS), Osteoporosis, Safety

\section{Citation:}

Watanabe, H., Sugiyama, K., Otsuji, N., Nakano, K., Arifuku, H., Wakayama, T., Tokita, S., Koyama, K., Hirata, H., Arima, M., Kurasawa, K., Fukushima, Y. (2023) Effect of inhaled corticosteroids on bone mineral density in patients with asthma. Asian Pac J Allergy Immunol, 41(1), 45-52. https://doi.org/10.12932/ap-191019-0663

\footnotetext{
Affiliations:

${ }^{1}$ Department of Respiratory Medicine and Clinical Immunology Dokkyo Medical University, Saitama Medical Center, Japan

${ }^{2}$ Department of Rheumatology, Dokkyo Medical University, Japan

${ }^{3}$ National Hospital Organization, Utsunomiya National Hospital, Japan
}

Corresponding author:

Kumiya Sugiyama

Department of Respiratory Medicine and Clinical Immunology

Dokkyo Medical University, Saitama Medical Center

2-1-50 Minami-Koshigaya, Koshigaya, Saitama 343-8555, Japan

E-mail: sugiyama@dokkyomed.ac.jp

\section{Introduction}

Inhaled corticosteroids (ICS) have been reported to reduce the risk of fatal asthma, with improved quality of life for patients. ${ }^{1-3}$ Serious side effects following the use of ICS are rare, unlike oral corticosteroids, and budesonide is an ICS that can be used safely for asthma in pregnancy. ${ }^{4}$ Thus, ICS use has greater beneficial effects for asthma and lower side effects for patients in general.

However, certain side effects have been reported for high doses of ICS. One such side effect is suppression of the hypothalamic-pituitary-adrenal axis. A case report on patients with asthma described acute adrenal crisis caused by high doses of fluticasone propionate. ${ }^{5}$ Also, high doses of both fluticasone propionate and budesonide in patients with asthma were reported to significantly decrease 24 -h urine cortisol excretion and suppress serum cortisol levels. ${ }^{6,7}$ In addition, high doses of both chlorofluorocarbon-beclomethasone dipropionate and hydrofluoroalkane-134a beclomethasone dipropionate (HFA-BDP) for patients with asthma resulted 
in significantly lower 24-h urinary free cortisol excretion than with placebo. ${ }^{8}$ In contrast, lower doses of fluticasone propionate, budesonide, DFD-BDP, and HFA-BDP had no effect on the hypothalamic-pituitary-adrenal axis in patients with asthma. ${ }^{6-9}$ Although the dose and potential duration of ICS therapy will be important in terms of suppression of the hypothalamic-pituitary-adrenal axis, the actual suppressive dose of ICS has not been determined.

The prevalence of osteoporosis in patients asthma was reported to be the same as that in those without asthma and was not increased. ${ }^{10}$ In addition, high doses of fluticasone propionate had no effects on bone mineral density (BMD) from baseline at 2 years. ${ }^{11}$ However, in other studies, asthma was associated with clinically significant BMD decrease, ${ }^{12}$ and a negative correlation was seen between total cumulative dose of ICS and BMD in patients with asthma. ${ }^{13}$ Furthermore, ICS in childhood may have potential adverse effects on growth velocity; ${ }^{14-16}$ therefore, ICS may have potentially harmful effects on bone metabolism in addition to suppressing the hypothalamic-pituitary-adrenal axis.

These discrepant results suggest that systemic corticosteroids may contribute to osteoporosis because they are typically used to treat exacerbation of asthma. ${ }^{17}$ Also, the duration of ICS use, but not daily dose of ICS, will have a greater effect on osteoporosis in patients with asthma. This study sought to determine the effects of ICS use on BMD in patients with asthma, by analyzing the relationship between BMD and lifetime cumulative ICS dose for treatment durations of over 6 years on average.

\section{Materials and Methods Study design}

Subjects were prospectively enrolled for this study. To evaluate bone metabolism in ICS, we evaluated the relationship between lifetime cumulative ICS dose and bone density. We calculated lifetime cumulative ICS dose from all past prescriptions. For comparison of different ICSs, fluticasone propionate was used a standard and the dose of other ICSs was calculated relative to the dose of fluticasone propionate. ${ }^{18}$ When the glucocorticoid receptor-binding affinity of dexamethasone was defined as 100 , the affinities of fluticasone propionate, budesonide, beclomethasone dipropionate, ciclesonide, and fluticasone furoate were 1775, $855,1345,1212$, and 2989, respectively. According to the above calculation, $100 \mu \mathrm{g}$ of budesonide is equivalent to $48.2 \mu \mathrm{g}$ of fluticasone propionate. Thus, we evaluated the correlation between lifetime cumulative ICS dose and bone density.

In a sub-analysis, we evaluated the differences in effects on bone density between fluticasone propionate and budesonide, which are used widely in Japan. Where patients had used 2 or more ICSs over a lifetime, patients for whom the lifetime cumulative ICS dose comprised over $80 \%$ of either fluticasone propionate or budesonide were enrolled in the fluticasone propionate or budesonide group, respectively.

This study was approved by the ethics committee of Dokkyo Medical University Saitama Medical Center (No. 1553). Written informed consent was obtained from all patients.

\section{Subjects}

Subjects were patients with stable asthma who visited to our hospital regularly and were receiving ICS for asthma, but without regular oral corticosteroids. We excluded patients receiving treatment for osteoporosis and/or supplementation of calcium and/or vitamin D. Those with complications or risk factors for osteoporosis, such as rheumatoid arthritis, diabetes mellitus, chronic kidney disease, and COPD were also excluded. However, patients with a history of transient use of oral corticosteroids and/or corticosteroid infusion for exacerbation of asthma were not excluded. In total, 40 patients (14 men, 26 women; mean age 61.2 years) were enrolled. Baseline characteristics of the patients are shown in Table 1. Basal doses of ICS were calculated from real doses of budesonide, fluticasone furoate, and ciclesonide to a standard dose of fluticasone propionate. Systemic corticosteroids were used to treat exacerbation of asthma with $2.5 \mathrm{mg}$ prednisolone as a standard dose. One patient required Step 5 treatment according to the Global Initiative for Asthma 2018 classification, because the patient was receiving omalizumab without oral corticosteroids. ${ }^{19}$ No significant differences were observed in baseline characteristics between budesonide and fluticasone propionate, except duration and basal doses of ICS.

\section{Measurement of bone density and markers of bone metabolism}

Bone density in the radius was measured using the LD-100 ultrasonic bone densitometry system (Oyo Electric Co., Ltd., Kyoto, Japan). The LD-100 measures three kinds of bone density in the radius, namely, whole bone density, cortical bone thickness, and cancellous bone density. ${ }^{20-22}$ It measures the propagation speeds of fast and slow waves in the radius, and the attenuation $(\mathrm{dB})$ of these waves gives whole bone density in the radius. For ease of understanding, attenuation in the young adult mean (YAM) or the age-matched mean (AM) were set as $100 \%{ }^{23}$ Cortical bone thickness $(\mathrm{mm})$ and cancellous bone density $\left(\mathrm{mg} / \mathrm{cm}^{3}\right)$ are calculated using these parameters. Bone density was measured at least three times in different months and the means of the values were calculated.

We also measured bone metabolism markers in serum including bone-specific alkaline phosphatase (BAP), tartrate-resistant acid phosphatase 5b (TRACP-5b), whole parathyroid hormone (PTH), and N-terminal telopeptide (NTx).

\section{Statistical analysis}

All statistical analysis was performed using Microsoft ${ }^{\oplus}$ Excel $^{\circledR} 2016$ MSO (Microsoft Corp., Redmond, WA) and $\mathrm{JMP}^{\circledast}$ Pro version 11.0.0 (SAS institute, Cary, NC) statistical software. Differences between two independent samples were examined using the chi-square test and Mann-Whitney U test. Relationships between two parameters were examined using correlation coefficients and linear regression analysis. Differences at $p<0.05$ were considered significant. Results are expressed as mean \pm standard deviation. 
Table 1. Patient baseline characteristics

\begin{tabular}{|c|c|c|c|}
\hline & Total $^{*}$ & Fluticasone propionate group & Budesonide group \\
\hline Patient (n) & 40 & 15 & 14 \\
\hline Mean age (years) & $61.2 \pm 12.6$ & $62.1 \pm 11.8$ & $60.0 \pm 14.5$ \\
\hline Male/Female & $14 / 26$ & $6 / 9$ & $3 / 11$ \\
\hline Duration $\left(\right.$ years) ${ }^{*}$ & $6.19 \pm 6.05$ & $8.11 \pm 6.69$ & $2.84 \pm 2.39$ \\
\hline \multicolumn{4}{|l|}{ Severity } \\
\hline Step II & 2 & 1 & 1 \\
\hline Step III & 7 & 3 & 3 \\
\hline Step IV & 30 & 11 & 10 \\
\hline Step V & 1 & 0 & 0 \\
\hline FeNO (ppb) & $29.8 \pm 20.6$ & $33.3 \pm 21.6$ & $27.5 \pm 26.0$ \\
\hline \multicolumn{4}{|l|}{ Pulmonary Function } \\
\hline$\% \mathrm{FVC}$ & $102.5 \pm 18.1$ & $100.5 \pm 22.9$ & $106.5 \pm 14.9$ \\
\hline$\% \mathrm{FEV}_{1}$ & $91.3 \pm 19.7$ & $92.4 \pm 23.4$ & $94.3 \pm 15.3$ \\
\hline $\mathrm{FEV}_{1} \%$ & $69.9 \pm 10.6$ & $72.1 \pm 11.6$ & $70.0 \pm 8.72$ \\
\hline$\% \mathrm{~V}_{50}$ & $51.4 \pm 29.6$ & $59.6 \pm 31.0$ & $49.9 \pm 30.6$ \\
\hline$\% \mathrm{~V}_{25}$ & $35.4 \pm 22.5$ & $45.6 \pm 27.9$ & $30.3 \pm 13.8$ \\
\hline Basal dose of ICS $(\mu \mathrm{g} / \text { day })^{*}$ & $305.6 \pm 181.6^{\# \#}$ & $399.6 \pm 232.0$ & $217.9 \pm 83.9^{\# \#}$ \\
\hline Systemic corticosteroids (mg/month) & $2.5 \pm 8.5$ & $0.3 \pm 1.3$ & $0.7 \pm 1.8$ \\
\hline \multicolumn{4}{|l|}{ Concomitant drugs } \\
\hline LABA & 37 & 14 & 12 \\
\hline LTRA & 22 & 8 & 7 \\
\hline Theophylline & 10 & 5 & 2 \\
\hline
\end{tabular}

Mean \pm standard deviation $(\mathrm{SD}) .{ }^{\star} P<0.01$.

\#Total means all ICSs, including beclomethasone dipropionate, ciclesonide and fluticasone furoate.

\#Fluticasone propionate was used as a standard relative to which the dose of other ICSs was calculated.

\#\# Prednisolone was used as a standard relative to which the dose of other corticosteroids was calculated.

FeNO, Fractional exhaled nitric oxide; $\% \mathrm{FVC}$, forced vital capacity ( $\%$ of predicted); $\% \mathrm{FEV}_{1}$, forced expiratory volume in $1 \mathrm{~s}\left(\%\right.$ of predicted); $\% \mathrm{~V}_{50}$, maximum expiratory flow at $50 \%$ of the forced vital capacity ( $\%$ of predicted); $\% \mathrm{~V}_{25}$, maximum expiratory flow at $25 \%$ of the forced vital capacity ( $\%$ of predicted); LABA,

Long-acting beta-adrenoceptor agonist; LTRA, leukotriene receptor antagonist.

\section{Results}

Relationship between bone density and lifetime cumulative ICS dose

The whole bone density, cortical bone thickness, and cancellous bone density of the radius are shown in Figure 1A, 1B, and 1C, respectively. Bone density in YAM was set as $100 \%$. Mean whole bone density was $90.8 \pm 11.2 \%$, cortical bone thickness mean was $3.43 \pm 0.90 \mathrm{~mm}$, and mean cancellous bone density was $164 \pm 50 \mathrm{mg} / \mathrm{cm}^{3}$. Bone density and lifetime ICS dose showed no significant correlation with whole bone density $\left(\mathrm{Y}=-3.4 * 10^{-3} \mathrm{X}+93.2, \mathrm{r}^{2}=0.069\right)$, cortical bone thickness $\left(\mathrm{Y}=-0.3 * 10^{-3} \mathrm{X}+3.6, \mathrm{r}^{2}=0.093\right)$, and cancellous bone density $\left(\mathrm{Y}=-13.5 * 10^{-3} \mathrm{X}+173.0\right.$, $\left.r^{2}=0.053\right)$.

To remove the effects of aging, whole bone density, cortical bone thickness and cancellous bone density in AM were set as $100 \%$. These were calculated and the values are shown Figure 2A, 2B, and 2C, respectively. Mean whole bone density was $100 \pm 13 \%$, mean cortical bone thickness was $100 \pm 21 \%$, and mean cancellous bone density was $99 \pm 31 \%$. Similarly, bone density and lifetime cumulative ICS dose showed no significant correlations in whole bone density $\left(\mathrm{Y}=-1.6^{*} 10^{-3} \mathrm{X}+101.4, \mathrm{r}^{2}=0.011\right)$, cortical bone thickness $\left(\mathrm{Y}=-3.7 * 10^{-3} \mathrm{X}+103.0, \mathrm{r}^{2}=0.022\right)$, and cancellous bone density $\left(\mathrm{Y}=-2.3 * 10^{-3} \mathrm{X}+100.9, \mathrm{r}^{2}=0.004\right)$. In the relationship between lower $(<300 \mathrm{mg})$, middle $(\geq 300$ $\mathrm{mg}$ and $<600 \mathrm{mg})$ and higher $(\geq 600 \mathrm{mg})$ lifetime cumulative ICS dose, no significant differences were observed between lower, middle, and/or higher lifetime cumulative ICS dose in whole bone density $(104.1 \pm 12.8 \%, 98.8 \pm 12.2 \%$, and 96.9 $\pm 13.7 \%$ : Figure 3A), cortical bone thickness (102.7 $\pm 19.8 \%$, $98.8 \pm 20.4 \%$, and $99.2 \pm 24.4 \%$ : Figure $3 B$ ), and cancellous bone density (106.0 $\pm 30.6 \%$, $98.6 \pm 32.7 \%$, and $91.2 \pm 30.8 \%$, respectively; Figure 3C). 
A

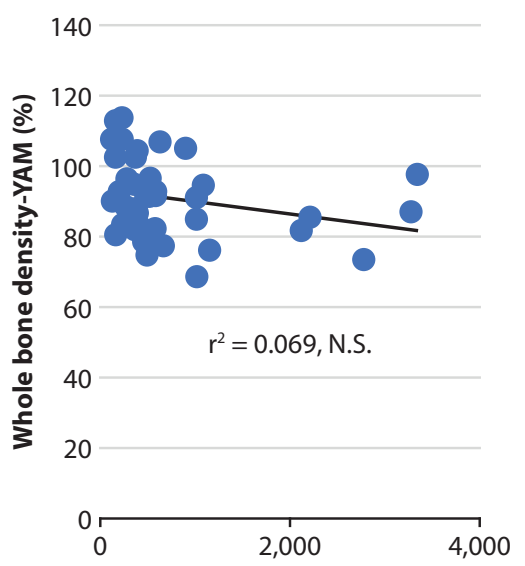

B

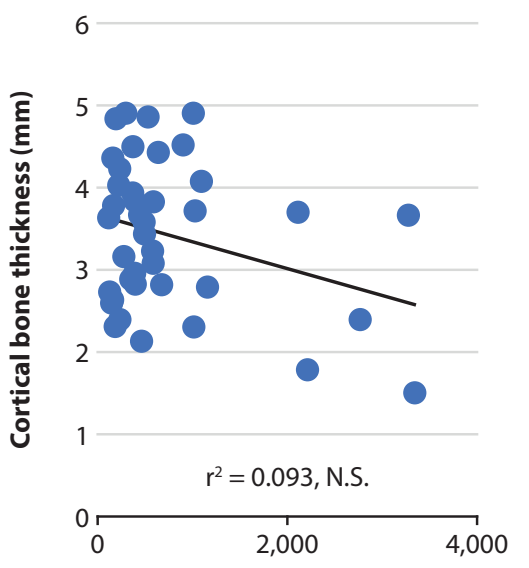

C

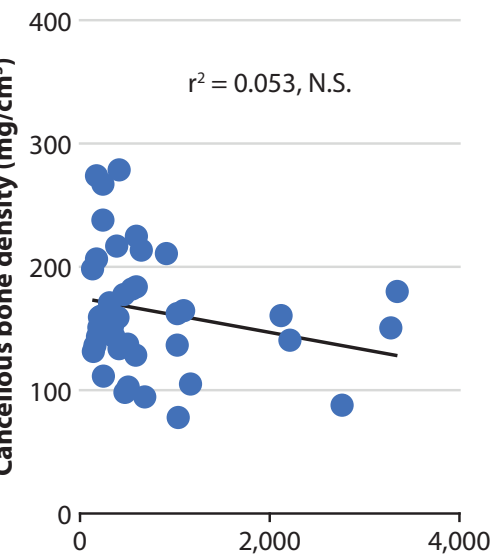

Total dose of inhaled corticosteroids $(\mathrm{mg})$

Figure 1. Relationship between lifetime cumulative ICS dose and whole bone density, cortical bone thickness, and cancellous bone density of the radius (A, B, and C, respectively). For whole bone density of the radius (A), young adult mean was set as $100 \%$. No significant negative correlations were observed.

A

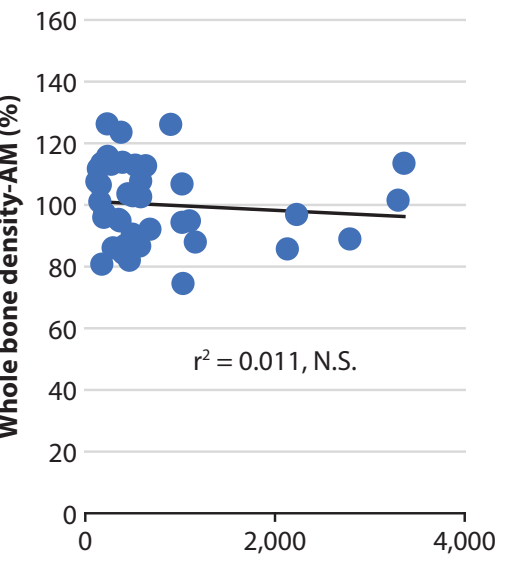

B

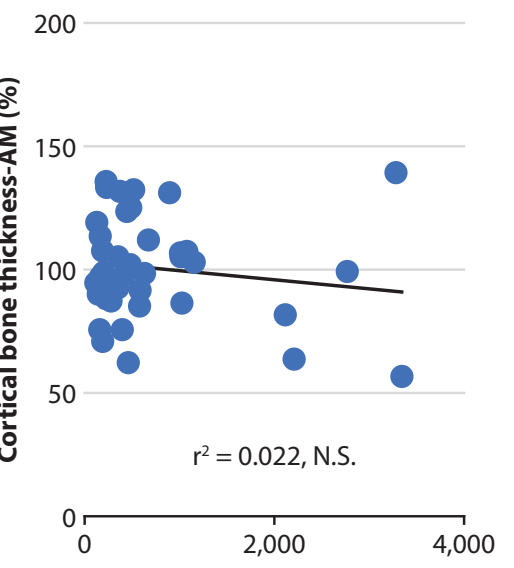

C

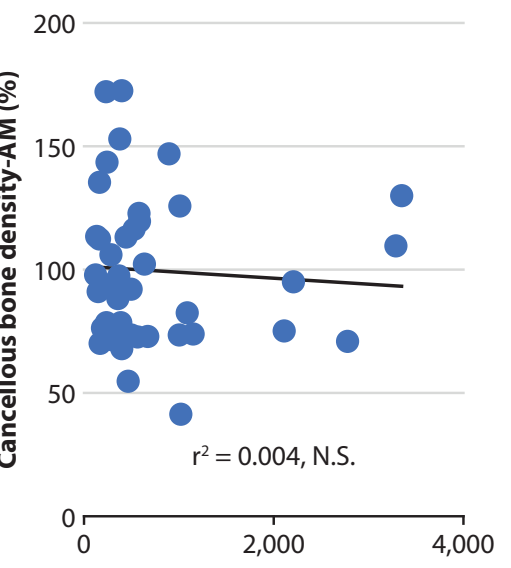

Total dose of inhaled corticosteroids ( $\mathrm{mg})$

Figure 2. Relationship between lifetime cumulative ICS dose and whole bone density, cortical bone thickness, and cancellous bone density of the radius (A, B, and C, respectively). For bone density, age-matched means were set as $100 \%$. No significant negative correlations were observed.

A

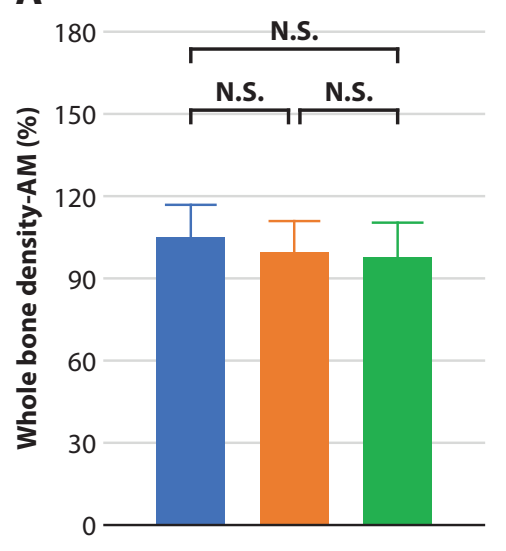

Lifetime cumulative dose of inhaled corticosteroids
B

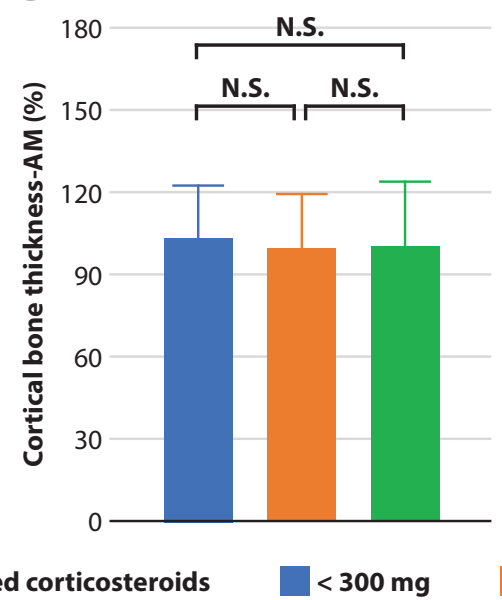

C

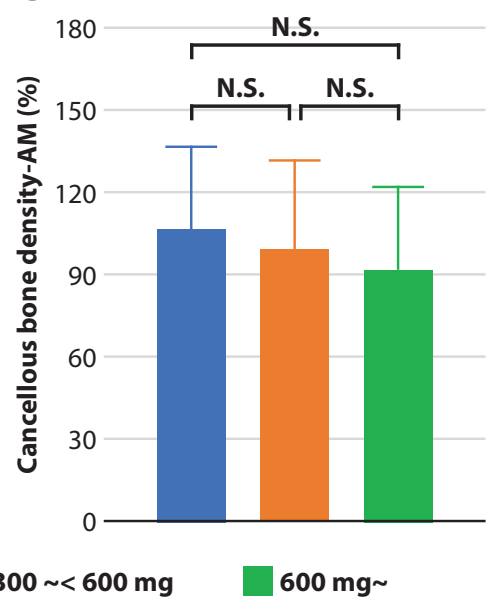

Figure 3. Relationship between lifetime cumulative ICS dose and whole bone density, cortical bone thickness, and cancellous bone density of the radius (A, B, and C, respectively). For bone density, age-matched means were set as $100 \%$. No significant differences were observed. 


\section{Effects of bone density on asthma severity}

To examine the relationship between asthma severity and bone density, the bone density in $\mathrm{AM}$ was set as $100 \%$ to remove the effects of aging.

The relationship between BMD and asthma severity is shown in Figure 4. Two combined groups designated Step $2 / 3$ and Step $4 / 5$ were evaluated, because of the small number of patients in Step 2 and 5. Mean whole bone density of the radius in Step 2/3 and Step 4/5 was $98 \pm 13 \%$ and $101 \pm 13 \%$, respectively. Mean cortical bone thickness and mean cancellous bone density was $105 \pm 15 \mathrm{~mm}$ and $99 \pm 22 \mathrm{~mm}$, respectively. Cancellous bone density was $92 \pm 32 \mathrm{mg} / \mathrm{cm}^{3}$ and $101 \pm 31 \mathrm{mg} / \mathrm{cm}^{3}$, respectively. No significant differences were observed between Step 2/3 and Step $4 / 5$ in all types of bone density.
A

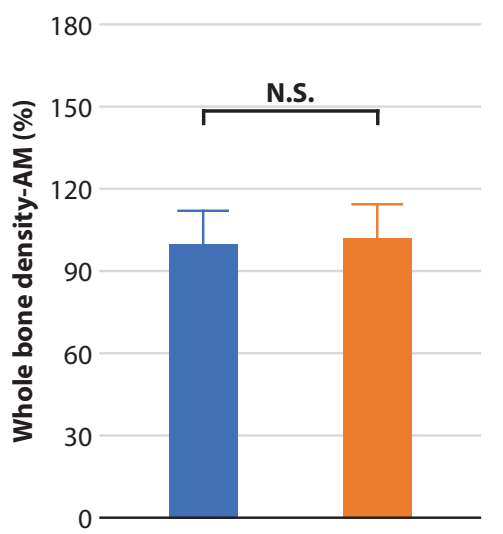

B 180

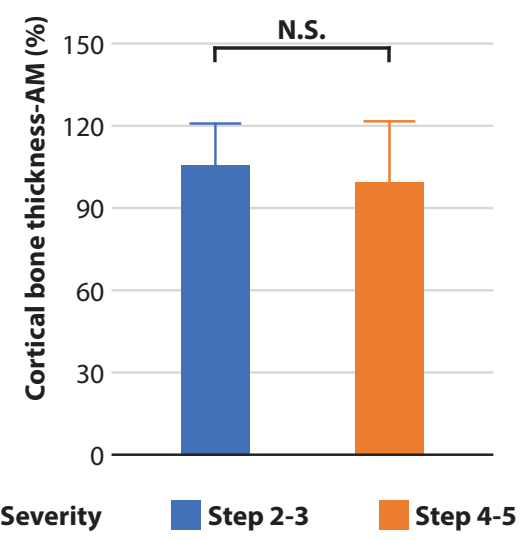

The analysis with asthma duration is shown in Figure 5. In the relationship between short $(<4$ years $)$, middle ( $\geq 4$ years and $<8$ years), and higher ( $\geq 8$ years) duration, no significant correlations were observed between lower and higher lifetime cumulative ICS dose in whole bone density $(102.8 \pm 11.4 \%, 99.8 \pm 16.8 \%$, and $96.8 \pm 9.8 \%$ : Figure 5A), cortical bone thickness $(102.0 \pm 18.3 \%, 101.4 \pm 22.9 \%$, and $96.6 \pm 24.2 \%$ : Figure 5B), and cancellous bone density $(108.5 \pm 32.1 \%, 95.8 \pm 35.1 \%$, and $88.4 \pm 20.1 \%$, respectively; Figure 5C). No significant correlations were observed with whole bone density $\left(\mathrm{Y}=-0.4 \mathrm{X}+102.8, \mathrm{r}^{2}=0.033\right)$, cortical bone thickness $\left(\mathrm{Y}=-0.9 \mathrm{X}+106.1, \mathrm{r}^{2}=0.069\right)$, and cancellous bone density $\left(\mathrm{Y}=-1.0 \mathrm{X}+105.4, \mathrm{r}^{2}=0.036\right)$.
Figure 4. Relationship between asthma severity and whole bone density, cortical bone thickness, and cancellous bone density of the radius (A, B and C, respectively). For bone density, age-matched means were set as $100 \%$. No significant differences were observed.
A

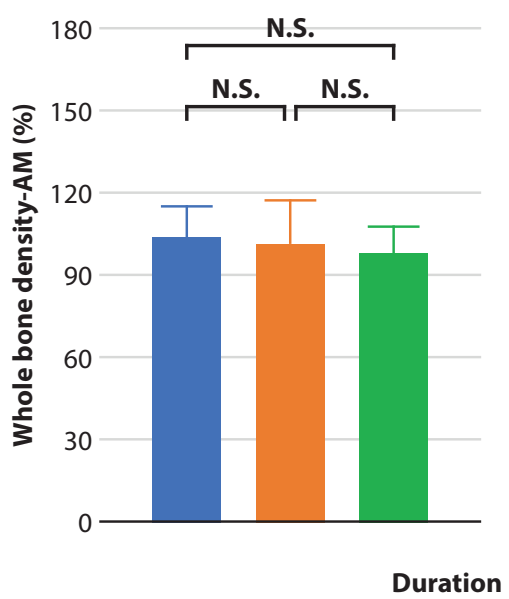

B

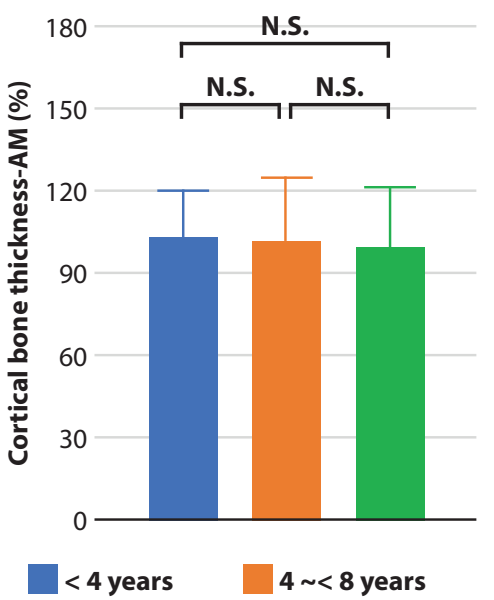

C

180

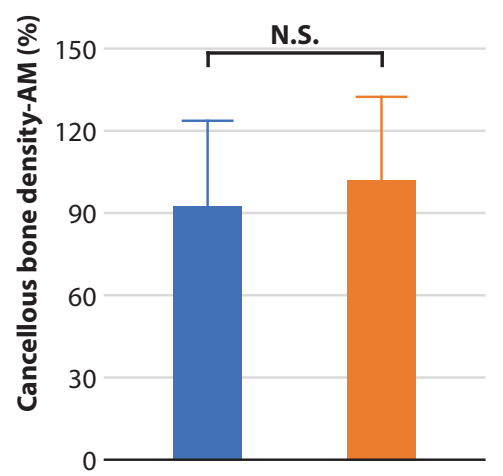

C

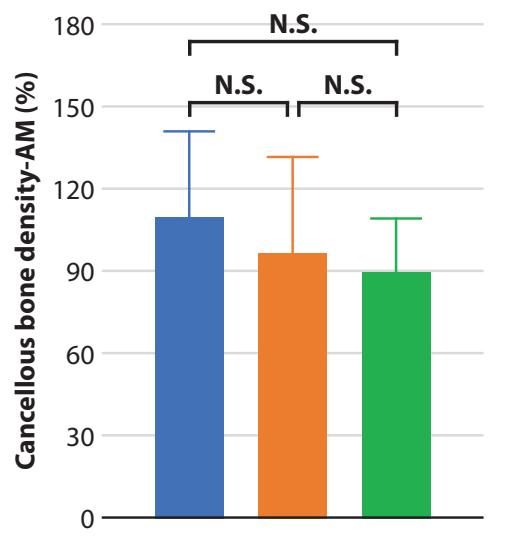

8 years

Figure 5. Relationship between asthma duration and whole bone density, cortical bone thickness, and cancellous bone density of the radius (A, B, and C, respectively). For bone density, age-matched means were set as $100 \%$. No significant differences were observed. 
In the analysis with pulmonary function, no significant differences were observed between $<100 \%$ and $\geq 100 \%$ forced vital capacity (\%FVC: \% of predicted) in whole bone density $(104.9 \pm 14.5 \%$ and $99.2 \pm 11.8 \%)$, cortical bone thickness $(107.1 \pm 23.9 \%$ and $96.8 \pm 20.5 \%)$, and cancellous bone density (108.2 $\pm 33.4 \%$ and $97.4 \pm 31.4 \%$, respectively). Although the standard value for $\% \mathrm{FVC}$ is $>80 \%$, we divided the values for the 2 groups by $100 \%$, because mean $\% \mathrm{FVC}$ was $103 \%$ and only a few patients had $\%$ FVC $<80 \%$. Also, $\% \mathrm{FVC}$ was not significantly correlated with whole bone density $\left(\mathrm{Y}=-6.5 * 10^{-2} \mathrm{X}+107.9, \mathrm{r}^{2}=0.008\right)$, cortical bone thickness $\left(\mathrm{Y}=-1.5 * 10^{-2} \mathrm{X}+102.0, \mathrm{r}^{2}<0.001\right)$, and cancellous bone density $\left(\mathrm{Y}=-1.4^{\star} 10^{-1} \mathrm{X}+115.8, \mathrm{r}^{2}=0.006\right)$. No significant differences were observed between $<90 \%$ and $\geq 90 \%$ Forced expiratory volume in $1 \mathrm{~s} \quad\left(\% \mathrm{FEV}_{1}\right.$ : $\%$ of predicted) in whole bone density $(101.4 \pm 14.3 \%$ and $101.1 \pm 12.3 \%)$, cortical bone thickness (102.1 $\pm 24.1 \%$ and $99.4 \pm 21.0 \%)$, and cancellous bone density (100.2 $\pm 33.9 \%$ and $102.1 \pm 31.5 \%$, respectively). Although the standard value for $\% \mathrm{FEV}_{1}$ is $>80 \%$, we divided the values for the 2 groups by $90 \%$, because mean $\% \mathrm{FEV}_{1}$ was $91 \%$. Also, $\% \mathrm{FEV}_{1}$ was not significantly correlated with whole bone density $\left(\mathrm{Y}=5.4^{*} 10^{-2} \mathrm{X}+96.3, \mathrm{r}^{2}=0.007\right)$, cortical bone thickness $\left(\mathrm{Y}=-5.4{ }^{*} 10^{-2} \mathrm{X}+105.5, \mathrm{r}^{2}=0.002\right)$, and cancellous bone density $\left(\mathrm{Y}=1.4^{\star} 10^{-1} \mathrm{X}+88.5, \mathrm{r}^{2}=0.007\right)$.

\section{Bone metabolism markers in serum}

The mean levels of BAP, TRACP-5b, PTH, and NTx were $14.4 \pm 5.2 \mu \mathrm{g} / \mathrm{L}, 404 \pm 154 \mathrm{mU} / \mathrm{dL}, 29.6 \pm 5.2 \mathrm{pg} / \mathrm{mL}$, and 18.3 $\pm 7.5 \mathrm{nmolBCE} / \mathrm{L}$, respectively. No significant differences and correlations were observed in lifetime cumulative ICS dose (data not shown).

\section{Effects of different ICS agents on bone density}

Next, we evaluated the differences between fluticasone propionate and budesonide in terms of bone density and lifetime cumulative ICS dose. Patients for whom the lifetime cumulative ICS dose comprised $80 \%$ or more of either fluticasone propionate or budesonide were defined as the fluticasone propionate group or the budesonide group, respectively. Whole bone density, cortical bone thickness, and cancellous bone density in AM were set as $100 \%$. Bone density and lifetime cumulative ICS dose showed no significant correlations with the whole bone density $\left(r^{2}=0.016\right.$ and $\left.r^{2}=0.057\right)$, cortical bone thickness $\left(r^{2}<0.001\right.$ and $\left.r^{2}=0.061\right)$, and cancellous bone density $\left(r^{2}=0.002\right.$ and $\left.r^{2}=0.059\right)$ in the fluticasone propionate and budesonide groups, respectively. Also, no significant differences were observed between fluticasone propionate or budesonide in those BMDs (data not shown).

\section{Discussion}

In this study, we analyzed the relationship between lifetime cumulative ICS dose and BMD. We used the LD-100 ultrasonic bone densitometry system, although the dual energy X-ray absorptiometry (DEXA) is popular in Japan. Peripheral quantitative computed tomography (pQCT) is known to have high accuracy and the LD-100 has high accuracy comparable to that of pQCT. In addition to high accuracy, the LD-100 has the added benefit of obtaining measurements without radiation exposure. Therefore, we opted to use the LD-100 in our study. ${ }^{20-22}$ The LD-100 densitometry system measures ultrasonic wave attenuation $(\mathrm{dB})$ in the radius, from which the results are calculated and the percentages are shown with YAM set as $100 \% .^{20-24}$ Our results are shown as percentages in YAM for whole bone density, but not for ultrasonic wave attenuation $(\mathrm{dB})$, for ease of understanding.

We found negative correlations between lifetime cumulative ICS dose and bone density of the radius, but these were not significant (Figure 1). In another analysis, we observed a positive correlation between age and lifetime cumulative ICS dose, although this was also not significant. This suggests that patients who have used large doses of ICS over their lifetime were older in age. These negative correlations were thus dependent on aging and are not likely a resultant effect of ICS. Therefore, no significant correlation was seen between bone density in AM and lifetime cumulative ICS dose as shown in Figure 2. Although it may be confusing to show the raw data in Figure 1, we think the raw data should be shown in a scientific study. Finally, use of ICS showed no effects on osteoporosis assessed by whole bone density, cortical bone thickness, and cancellous bone density over the 6-year observation period.

Several studies have described the relationship between ICS and BMD for patients with asthma. One report found no change in BMD at 2 years from values at baseline screening following $500 \mu \mathrm{g}$ twice daily fluticasone propionate. ${ }^{11}$ No statistically significant relationship was detected between the use of ICS and reduced BMD in older patients with asthma. ${ }^{11}$ In our study, we evaluated the lifetime cumulative ICS dose and found no effects of ICS on BMD, which is consistent with results from previous studies.

However, other studies have suggested that use of ICS reduced BMD. ${ }^{12,13,17}$ While ICS use in childhood may have potential adverse effects on growth velocity, ${ }^{14-16}$ the underlying mechanism may differ between BMD and growth velocity. This is because ICS use was not significantly associated with increased risk of fracture in a pediatric asthma population. ${ }^{25}$ Therefore, in the discrepant results on the relationship between ICS and osteoporosis, past medical history of systemic corticosteroids, but not ICS, may contribute to osteoporosis. Moreover, our study used low-dose systemic corticosteroids for exacerbation of asthma, and no significant differences were observed between no systemic corticosteroid group and with systemic corticosteroid group in those BMDs (data not shown). 
In addition, ICS use in childhood will likely have no effect on BMD. However, older patients with ACO using high ICS doses were found to be at increased risk of fracture. ${ }^{26}$ Although COPD is a risk factor for osteoporosis, female patients with COPD using ICSs have a protective dose-response effect for osteoporosis. ${ }^{27}$ The differential diagnosis between asthma and COPD is often difficult, and sometimes both asthma and COPD are present. In our study, patients with pure asthma and without COPD were enrolled, and based on the positive findings with regard to ICS and osteoporosis, underlying disease like COPD may contribute to osteoporosis, but not ICS. Although studies with large sample populations will have high reliability, it will be difficult to accumulate cases of distinct asthma without overlapping COPD. The number of subjects in our study was not so large, but we had patients with pure asthma without COPD, whose prescriptions from the onset of asthma were available. Because our data showed significant normal distribution by the Shapiro-Wilk test (data not shown), we consider the population size to be adequate for statistical analysis.

In our study, an ICS dose of $300 \mu \mathrm{g}$ fluticasone propionate used for over 6 years on average and severe asthma had no effects on BMD, although high doses of ICS have been reported to suppress the hypothalamic-pituitary-adrenal axis. $^{5-8}$ Thus, ICS use can be considered safe for asthma without risk of osteoporosis. However, smoking history is reported to be a risk factor for osteoporosis, and so patients with both asthma and a history of smoking should be monitored for changes in BMD.

\section{Conclusion}

In the entire study population, long-term ICS use was safe and was not associated with an increased risk of osteoporosis.

\section{Acknowledgments}

We thank Ms. Seiko Sekiguchi, Ms. Akiko Maejima, Ms. Natsumi Suzuki, and Mr. Kazunori Fukuda at Dokkyo Medical University Saitama Medical Center for technical assistance.

\section{Conflict of interest}

The authors declare that they have no conflict of interest.

\section{Source of funding}

This work was supported by Dokkyo Medical University.

\section{Authors contributions}

- HW, KS, and YF contributed to the conception and design of the study, the acquisition of data, and data analysis and interpretation.

- NO, KN, HA, TW, ST, and KK contributed to the conception and design of the study, the acquisition of data, and interpretation of the data.

- HH, MA, and KK contributed to the conception and design of the study and the interpretation of data.

- All authors read and approved the final manuscript.

\section{References}

1. Neffen H, Baena-Cagnani C, Passalacqua G, Canonica GW, Rocco D Asthma mortality, inhaled steroids, and changing asthma therapy in Argentina (1990-1999). Respir Med. 2006;100:1431-5.

2. Ebmeier S, Thayabaran D, Braithwaite I, Bénamara C, Weatherall M, Beasley R. Trends in international asthma mortality: analysis of data from the WHO Mortality Database from 46 countries (1993-2012). Lancet. 2017;390:935-45.

3. Tohda Y, Iwanaga T, Sano H, Kume H, Hirata K, Ohkura N, et al. Improved quality of life in asthma patients under long-term therapy: Assessed by AHQ-Japan. Int J Clin Pract. 2017; 71(1).

4. Norjavaara E, de Verdier MG. Normal pregnancy outcomes in a population-based study including 2968 pregnant women exposed to budesonide. J Allergy Clin Immunol 2003;111:736-42.

5. Todd GR, Acerini CL, Buck JJ, Murphy NP, Ross-Russell R, Warner JT, et al. Acute adrenal crisis in asthmatics treated with high-dose fluticasone propionate. Eur Respir J. 2002;19:1207-9.

6. Nielsen LP, Dahl R. Therapeutic ratio of inhaled corticosteroids in adult asthma. A dose-range comparison between fluticasone propionate and budesonide, measuring their effect on bronchial hyperresponsiveness and adrenal cortex function. Am J Respir Crit Care Med. 2000;162:2053-7.

7. Derom E, Van Schoor J, Verhaeghe W, Vincken W, Pauwels R. Systemic effects of inhaled fluticasone propionate and budesonide in adult patients with asthma. Am J Respir Crit Care Med. 1999;160:157-61.

8. Thompson PJ, Davies RJ, Young WF, Grossman AB, Donnell D. Safety of hydrofluoroalkane-134a beclomethasone dipropionate extrafine aerosol. Respir Med. 199;92 Suppl A:33-9.

9. Li JT, Goldstein MF, Gross GN, Noonan MJ, Weisberg S, Edwards L, et al. Effects of fluticasone propionate, triamcinolone acetonide, prednisone, and placebo on the hypothalamic-pituitary-adrenal axis. J Allergy Clin Immunol. 1999;103:622-9.

10. Katsura H, Kida K. A comparison of bone mineral density in elderly female patients with COPD and bronchial asthma. Chest. 2002;122: 1949-55.

11. Li JT, Ford LB, Chervinsky P, Weisberg SC, Kellerman DJ, Faulkner KG, et al. Fluticasone propionate powder and lack of clinically significant effects on hypothalamic-pituitary-adrenal axis and bone mineral density over 2 years in adults with mild asthma. J Allergy Clin Immunol. 1999;103:1062-8.

12. Jung JW, Kang HR, Kim JY, Lee SH, Kim SS, Cho SH. Are asthmatic patients prone to bone loss? Ann Allergy Asthma Immunol. 2014; 112:426-31.

13. Wong CA, Walsh LJ, Smith CJ, Wisniewski AF, Lewis SA, Hubbard R. Inhaled corticosteroid use and bone-mineral density in patients with asthma. Lancet. 2000;355:1399-403.

14. Kelly HW, Sternberg AL, Lescher R, Fuhlbrigge AL, Williams P, Zeiger RS, et al; CAMP Research Group. Effect of inhaled glucocorticoids in childhood on adult height. N Engl J Med. 2012;367:904-12.

15. Loke YK, Blanco $\mathrm{P}$, Thavarajah $\mathrm{M}$, Wilson AM. Impact of Inhaled Corticosteroids on Growth in Children with Asthma: Systematic Review and Meta-Analysis. PLoS One. 2015;10:e0133428. 
16. Philip J. The effects of inhaled corticosteroids on growth in children. Open Respir Med J. 2014;8:66-73.

17. Aljubran SA, Whelan GJ, Glaum MC, Lockey RF. Osteoporosis in the at-risk asthmatic. Allergy. 2014;69:1429-39.

18. Derendorf H, Meltzer EO. Molecular and clinical pharmacology of intranasal corticosteroids: clinical and therapeutic implications. Allergy. 2008;63:1292-300.

19. ginasthma.org [Internet]. Fontana: Global Initiative for Asthma; c2016 [cited 2016 Oct 10]. Available from: www.ginasthma.org

20. Otani, T. Quantitative Estimation of Bone Density and Bone Quality Using Acoustic Parameters of Cancellous Bone for Fast and Slow Waves. Jpn J Appl Phys. 2005;44:07GK05-1-07GK05-5.

21. Otani T, Mano I, Tsujimoto T, Yamamoto T, Teshima R, Naka H. Estimation of in vivo cancellous bone elasticity. Jpn J Appl Phys. 2009;48: 4578-82.

22. Sai $\mathrm{H}$, Iguchi $\mathrm{G}$, Tobimatsu $\mathrm{T}$, Takahashi $\mathrm{K}$, Otani $\mathrm{T}$, Horii $\mathrm{K}$, et al. Novel ultrasonic bone densitometry based on two longitudinal waves: significant correlation with pQCT measurement values and age-related changes in trabecular bone density, cortical thickness, and elastic modulus of trabecular bone in a normal Japanese population. Osteoporos Int. 2010; 21:1781-90.
23. Mano I, Horii K, Takai S, Suzaki T, Nagaoka H, Otani T. Development of Novel Ultrasonic Bone Densitometry Using Acoustic Parameters of Cancellous Bone for Fast and Slow Waves. Jpn J Appl Phys. 2006;45: 4700-2.

24. Yamamoto $\mathrm{T}$, Otani $\mathrm{T}$, Hagino $\mathrm{H}$, Katagiri $\mathrm{H}$, Okano $\mathrm{T}$, Mano $\mathrm{I}$, Teshima R. Measurement of human trabecular bone by novel ultrasonic bone densitometry based on fast and slow waves. Osteoporos Int. 2009; 20:1215-24.

25. Gray N, Howard A, Zhu J, Feldman LY, To T. Association between inhaled corticosteroid use and bone fracture in children with asthma. JAMA Pediatr. 2018;172:57-64.

26. Chan V, Cave AJ, Banh HL. Self-reported osteoporosis prevention in inhaled corticosteroid users in community pharmacy setting. SAGE Med Open. 2015; 3: 2050312115586912.

27. Liu SF, Kuo HC, Liu GH, Ho SC, Chang HC, Huang HT, et al. Inhaled corticosteroids can reduce osteoporosis in female patients with COPD. Int J Chron Obstruct Pulmon Dis. 2016;11:1607-14. 